\title{
STRICTNESS OF THE LOG-CONCAVITY OF GENERATING POLYNOMIALS OF MATROIDS
}

\author{
SATOSHI MURAI, TAKAHIRO NAGAOKA, AND AKIKO YAZAWA
}

\begin{abstract}
Recently, it was proved by Anari-Oveis Gharan-Vinzant, Anari-LiuOveis Gharan-Vinzant and Brändén-Huh that, for any matroid $M$, its basis generating polynomial and its independent set generating polynomial are log-concave on the positive orthant. Using these, they obtain some combinatorial inequalities on matroids including a solution of strong Mason's conjecture. In this paper, we study the strictness of the log-concavity of these polynomials and determine when equality holds in these combinatorial inequalities. We also consider a generalization of our result to morphisms of matroids.
\end{abstract}

\section{INTRODUCTION}

Given a matroid $M$ on $[n]=\{1,2, \ldots, n\}$ of rank $r$, one can associate two important polynomials called the basis generating polynomial and the independent set generating polynomial. The basis generating polynomial of $M$ is the polynomial

$$
f_{M}=\sum_{B \in \mathcal{B}(M)}\left(\prod_{i \in B} x_{i}\right) \in \mathbb{Z}\left[x_{1}, \ldots, x_{n}\right],
$$

where $\mathcal{B}(M)$ is the set of bases of $M$. The independent set generating polynomial of $M$ is the polynomial

$$
P_{M}=\sum_{I \in \mathcal{I}(M)}\left(\prod_{i \in I} x_{i}\right) x_{0}^{n-|I|} \in \mathbb{Z}\left[x_{0}, x_{1}, \ldots, x_{n}\right],
$$

where $\mathcal{I}(M)$ is the set of independent sets of $M$ and where $|X|$ denotes the cardinality of a finite set $X$. It is also useful to consider the polynomial $\bar{P}_{M}:=\left(\frac{\partial}{\partial x_{0}}\right)^{n-r} P_{M}$, which we call the reduced independent set generating polynomial of $M$.

These polynomials catch interest of many researchers recently and have been actively studied from combinatorial and algebraic point of view. See e.g. [AOV, ALOVI, ALOVII, BH1, BH2, COSW, EH, MN, NY, Ya]. Let $H_{f}=\left(\frac{\partial}{\partial x_{i}} \frac{\partial}{\partial x_{j}} f\right)$ be the Hessian matrix of a polynomial $f$. It was proved by Anari-Oveis GharanVinzant [AOV], Anari-Liu-Oveis Gharan-Vinzant [ALOVI, ALOVII] and BrändénHuh [BH1, BH2] that $f_{M}, P_{M}$ and $\bar{P}_{M}$ are log-concave on the positive orthant, equivalently, the Hessian matrix $\left.H_{f_{M}}\right|_{\boldsymbol{x}=\boldsymbol{a}}$ (resp. $\left.H_{P_{M}}\right|_{\boldsymbol{x}=\boldsymbol{a}}$ and $\left.H_{\bar{P}_{M}}\right|_{\boldsymbol{x}=\boldsymbol{a}}$ ) has exactly one positive eigenvalue for any $\boldsymbol{a} \in \mathbb{R}_{>0}^{n}$ (resp. $\boldsymbol{a} \in \mathbb{R}_{>0}^{n+1}$ ). The log-concavity of these polynomials has important applications to combinatorial properties of matroid. Let $M$ be a matroid on $[n]$ of rank $r \geq 2$. We write $\mathcal{B}_{i}(M):=\{B \in \mathcal{B}(M) \mid i \in B\}$

2010 Mathematics Subject Classification. 05C31, 05B35, 13E10 .

Key words and phrases. matroid, independent set, Mason's conjecture, Lorentzian polynomial, Hodge-Riemann relation, morphism of matroids. 
and $\mathcal{B}_{i j}(M):=\{B \in \mathcal{B}(M) \mid\{i, j\} \subset B\}$. Also, we write $I_{k}(M)$ for the number of independent sets of size $k$ of $M$ and $\widetilde{I}_{k}(M)=I_{k}(M) /\left(\begin{array}{l}n \\ k\end{array}\right)$. The log-concavity of $f_{M}$ and $\bar{P}_{M}$ is known to imply the following combinatorial inequalities.

(*) $|\mathcal{B}(M)| \times\left|\mathcal{B}_{i j}(M)\right| \leq 2\left(1-\frac{1}{r}\right)\left|\mathcal{B}_{i}(M)\right| \times\left|\mathcal{B}_{j}(M)\right|$ for all $i, j \in[n]$;

(**) $\widetilde{I}_{k-1}(M) \times \widetilde{I}_{k+1}(M) \leq\left(\widetilde{I}_{k}(M)\right)^{2}$ for all $k \geq 1$.

(See [HSW, Theorem 5] and [HW, Remark 15] for $(*)$ and see [ALOVII, Theorem $1.2]$ and [BH1, Corollary 7] for $(* *)$.) Note that the latter inequality was known as strong Mason's conjecture.

The purpose of this paper is to study when $f_{M}$ and $\bar{P}_{M}$ are strictly log-concave, and determine when equality holds in $(*)$ and $(* *)$. Our main result is the following.

Theorem 1.1. Let $M$ be a simple matroid on $[n]$ of rank $r \geq 2$.

(i) The Hessian matrix $\left.H_{f_{M}}\right|_{\boldsymbol{x}=\boldsymbol{a}}$ has signature $(+,-, \ldots,-)$ for any $\boldsymbol{a} \in \mathbb{R}_{>0}^{n}$, in particular, $f_{M}$ is strictly log-concave on $\mathbb{R}_{>0}^{n}$.

(ii) If $M$ is not a uniform matroid, then $\left.H_{\bar{P}_{M}}\right|_{\boldsymbol{x}=\boldsymbol{a}}$ has signature $(+,-, \ldots,-)$ for any $\boldsymbol{a}=\left(a_{0}, a_{1}, \ldots, a_{n}\right) \in \mathbb{R}^{n+1}$ with $a_{0} \geq 0$ and $a_{1}, \ldots, a_{n}>0$.

Recall that, for a matroid $M$ on $[n]$, the girth of $M$ is the minimum cardinality of its circuit, equivalently, $\operatorname{girth}(M)=\min \left\{k \mid I_{k}(M) \neq\left(\begin{array}{l}n \\ k\end{array}\right)\right\}$. Theorem 1.1 gives the following combinatorial consequences relating $(*)$ and $(* *)$.

Corollary 1.2. Let $M$ be a (not necessary simple) matroid on $[n]$ of rank $\geq 2$.

(i) Let $i, j \in[n]$ be non-loops. Then $|\mathcal{B}(M)| \times\left|\mathcal{B}_{i j}(M)\right|=2\left(1-\frac{1}{r}\right)\left|\mathcal{B}_{i}(M)\right| \times$ $\left|\mathcal{B}_{j}(M)\right|$ if and only if $i$ and $j$ are not parallel and $M$ has exactly two parallel classes.

(ii) $\widetilde{I}_{k-1}(M) \times \widetilde{I}_{k+1}(M)=\left(\widetilde{I}_{k}(M)\right)^{2}$ if and only if $k+1<\operatorname{girth}(M)$.

The if part of the above corollary is easy. Indeed, if $M$ has exactly two parallel classes, then $r=2$ and $\mathcal{B}(M)=\{\{x, y\} \mid x \in X, y \in Y\}$ for some disjoint sets $X$ and $Y$, so $|\mathcal{B}(M)| \times\left|\mathcal{B}_{i j}(M)\right|=\left|\mathcal{B}_{i}(M)\right| \times\left|\mathcal{B}_{j}(M)\right|=|X| \times|Y|$ when $i \in X, j \in Y$. Also, if $k+1<\operatorname{girth}(M)$, then $\widetilde{I}_{k-1}(M)=\widetilde{I}_{k}(M)=\widetilde{I}_{k+1}(M)=1$. Note also that, if $i$ is a loop of $M$, then $\mathcal{B}_{i}(M)=\mathcal{B}_{i j}(M)=\emptyset$.

The strictness of the log-concavity of $f_{M}$ was studied by the second and the third author in their previous paper $[\mathrm{NY}]$, where the statement (i) was proved for graphic matroids using the theory of prehomogenous vector spaces. Our proof in this paper is based on relations between the strong Lefschetz property, the Hodge-Riemann relation, and the Lorentzian property introduced in [BH2].

This paper is organized as follows: In section 2, we discuss properties of matroids and their generating polynomials. In section 3, we discuss relations between the strong Lefschetz property, the Hodge-Riemann relation and the Lorentzian property. In section 4, we prove our main results. Finally, in section 5, we consider a generalization of Theorem 1.1 to morphism of matroids.

Acknowledgements. The authors wish to express their gratitude to Yasuhide Numata for fruitful discussions. The research of the first author is partially supported by KAKENHI 16K05102, and the research of the second author is partially supported by Grant-in-Aid for JSPS Fellows 19J11207. 


\section{Matroids AND Their GENERATING POLYNOMIALS}

We first introduce some notation and terminology on matroids. We refer the readers to $[\mathrm{Ox}]$ for basic properties of matroids. A matroid on $[n]$ is an ordered pair $M=([n], \mathcal{B}(M))$ consisting of finite set $[n]$ and a non-empty collection $\mathcal{B}(M)$ of subsets of $[n]$ satisfying the following property:

If $B_{1}, B_{2} \in \mathcal{B}(M)$ and $x \in B_{1} \backslash B_{2}$, then there is a $y \in B_{2} \backslash B_{1}$ such that $\left(B_{1} \backslash\{x\}\right) \cup\{y\} \in \mathcal{B}(M)$.

An element of $\mathcal{B}(M)$ is called a basis of $M$ and a subset of a basis of $M$ is called an independent set of $M$. We denote by $\mathcal{I}(M)$ the set of independent sets of $M$. It is known that each basis has the same cardinality. The rank of a subset $X \subset[n]$ in $M$ is the maximum of the cardinality of independent subsets in $X$ and is denoted by $\operatorname{rank} X$ or $\operatorname{rank}_{M} X$. For any subset $X \subset[n]$, we define its closure as $\langle X\rangle:=$ $\{i \in[n] \mid \operatorname{rank}(X \cup\{i\})=\operatorname{rank} X\}$. We call $F \subset[n]$ a flat of $M$ if $F=\langle F\rangle$. A subset of $[n]$ which is not an independent set is called a dependent set of $M$. A minimal dependent set of $M$ is called a circuit of $M$. A circuit having cardinality $k$ is called a $k$-circuit. In particular 1-circuit is called a loop. We call an element $e$ a coloop of $M$ if it is contained in each basis of $M$. Also, if two elements $e_{1}$ and $e_{2}$ form a 2-circuit, then we call $e_{1}$ and $e_{2}$ are parallel. We say that a matroid $M$ is loopless (resp. simple) if it has no loops (resp. no loops and no parallel elements).

Example 2.1. Let $\mathcal{B}$ be the collection of $r$-element subsets of $[n]$, where $r \leq n$. Then $([n], \mathcal{B})$ is a matroid of $\operatorname{rank} r$ denoted by $U_{r, n}$. This matroid is called the uniform matroid of rank $r$ on an $n$-element set. It is known and easily checked by definition that all rank 2 simple matroids are uniform matroids.

Let $M=([n], \mathcal{B}(M))$ be a matroid. For $e \in[n]$ which is not a loop of $M$, we define the matroid $M / e$ on $[n] \backslash\{e\}$ by $\mathcal{B}(M / e):=\{B \backslash\{e\} \mid e \in B \in \mathcal{B}(M)\}$, which is called the contraction of $M$ w.r.t. e. Also, for $X \subset[n]$, we define the matroid $\left.M\right|_{X}$ on $[n] \backslash X$ by $\mathcal{B}\left(\left.M\right|_{X}\right):=\{B \in \mathcal{I}(M)|B \subset X| B \mid,=\operatorname{rank}(X)\}$, which is called the restriction of $M$ to $X$. In particular, for $e \in[n]$, we write $M \backslash e=\left.M\right|_{[n] \backslash\{e\}}$ and call it the deletion of $e$ from $M$.

For a matroid $M$ on $[n]$, there is a unique partition $[n]=E_{0} \sqcup E_{1} \sqcup \cdots \sqcup E_{s}$, called the parallel class decomposition, such that $E_{0}$ consists of all loops and that $i, j \in[n]$ are parallel if and only if they belongs to the same $E_{k}$, where $\sqcup$ denotes a disjoint union. We call $E_{1}, \ldots, E_{s}$ parallel classes of $M$. Recall that, for a matroid $M=([n], \mathcal{B}(M))$, its simplification $\bar{M}$ is the matroid obtained from $M$ by deleting all loops and deleting all but one element in each parallel class in the matroid $M$. We also define the truncation $T M=([n], \mathcal{B}(T M))$ by $\mathcal{B}(T M)=\{I \in \mathcal{I}(M)|| I \mid=$ $\operatorname{rank}(M)-1\}$, and inductively define $T^{k} M:=T\left(T^{k-1} M\right)$ for $k>1$.

Below we write some obvious properties of basis generating polynomials and independent set generating polynomials. In the rest of this paper, we write $\partial_{i}=\frac{\partial}{\partial x_{i}}$.

Lemma 2.2. Let $M$ be a matroid on $[n]$ of rank $r$.

(i) If $i \in[n]$ is a loop, then $\partial_{i} f_{M}=\partial_{i} P_{M}=0$.

(ii) If $i \in[n]$ is not a loop, then $\partial_{i} f_{M}=f_{M / i}$ and $\partial_{i} P_{M}=P_{M / i}$. 
(iii) If $i_{1}, i_{2} \in[n]$ are parallel, then $\partial_{i_{1}} f_{M}=\partial_{i_{2}} f_{M}$ and $\partial_{i_{1}} P_{M}=\partial_{i_{2}} P_{M}$. Moreover, if $[n]=E_{0} \sqcup E_{1} \sqcup \cdots \sqcup E_{s}$ is the parallel class decomposition, then

$$
f_{M}=f_{\bar{M}}\left(\sum_{i \in E_{1}} x_{i}, \ldots, \sum_{i \in E_{s}} x_{i}\right)
$$

and

$$
P_{M}=x_{0}^{n-s} P_{\bar{M}}\left(x_{0}, \sum_{i \in E_{1}} x_{i}, \ldots, \sum_{i \in E_{s}} x_{i}\right),
$$

where $E_{0}$ is the set of loops and we consider that $\bar{M}$ is a matroid on $[s]$ such that $i$ corresponds to an element in $E_{i}$ for $i=1,2, \ldots, s$.

If the Hessian matrix of a polynomial $f \in \mathbb{R}\left[x_{1}, \ldots, x_{n}\right]$ is non-singular at some $\boldsymbol{a} \in \mathbb{R}^{n}$, then the polynomials $\partial_{1} f, \ldots, \partial_{n} f$ must be $\mathbb{R}$-linearly independent. In the rest of this section, to prove Theorem 1.1, we first prove this weaker property.

We need the following combinatorial property of flats of matroids. See [Ox, Section 1.4, Exercise 11].

Lemma 2.3. Let $M$ be a matroid on $[n]$ and $F$ a flat of $M$. If $\left\{G_{1}, \ldots, G_{\ell}\right\}$ is the set of minimal flats of $M$ that properly contain $F$, then $[n] \backslash F=\bigsqcup_{i=1}^{\ell}\left(G_{i} \backslash F\right)$.

Also, we often use the following elementary fact.

Lemma 2.4. Let $n \geq 2$ and $a_{0}, a_{1}, \ldots, a_{n} \in \mathbb{R}$. If $\sum_{j \neq k} a_{j}=a_{0}$ for all $k=$ $1,2, \ldots, n$, then $a_{1}=a_{2}=\cdots=a_{n}=\frac{1}{n-1} a_{0}$.

Proof. Let $J$ be the all 1 matrix of size $n$ and $E$ the identify matrix of size $n$. Then the matrix $J-E$ is non-singular and $\left(a_{1}, \ldots, a_{n}\right)$ must be the unique solution of the system of linear equations $(J-E) \cdot{ }^{t}\left(x_{1}, \ldots, x_{n}\right)={ }^{t}\left(a_{0}, a_{0}, \ldots, a_{0}\right)$.

The following is the main result of this section.

Theorem 2.5. Let $M$ be a simple matroid on $[n]$ of rank $r \geq 2$.

(i) $\partial_{1} f_{M}, \ldots, \partial_{n} f_{M}$ are $\mathbb{R}$-linearly independent.

(ii) If $M \neq U_{r, n}$ then $\partial_{0} \bar{P}_{M}, \partial_{1} \bar{P}_{M}, \ldots, \partial_{n} \bar{P}_{M}$ are $\mathbb{R}$-linearly independent.

Proof. (i) Suppose $\left(a_{1} \partial_{1}+\cdots+a_{n} \partial_{n}\right) f_{M}=0$ for some $a_{1}, \ldots, a_{n} \in \mathbb{R}$. We will prove $a_{1}=\cdots=a_{n}=0$. To do this, we actually prove the following statement using decent induction on the rank of flats.

$$
\sum_{j \in[n] \backslash F} a_{j}=0 \quad \text { for all flats } F \neq[n] \text { of } M .
$$

Note that (1) and Lemma 2.4 imply $a_{1}=\cdots=a_{n}=0$ since the equations for rank 1 flats tell $\sum_{j \neq k} a_{j}=0$ for all $k=1,2, \ldots, n$.

We first prove (1) when $F$ has rank $r-1$. Let $I \in \mathcal{I}(M)$ be an independent set such that $\langle I\rangle=F$. Then $|I|=r-1$ and the coefficient of $\prod_{i \in I} x_{i}$ in $\left(a_{1} \partial_{1}+\cdots+a_{n} \partial_{n}\right) f_{M}$ is

$$
\sum_{j \notin I,\{j\} \cup I \in \mathcal{B}(M)} a_{j}=\sum_{j \in[n] \backslash F} a_{j},
$$

which must be zero since we assume $\left(a_{1} \partial_{1}+\cdots+a_{n} \partial_{n}\right) f_{M}=0$.

Now suppose $F$ has rank $<r-1$ and assume that (1) holds for all flats $G$ that properly contain $F$. Let $G_{1}, \ldots, G_{\ell}$ be the minimal flats that properly contains $F$. 
Note that $\ell \geq 2$ since, by Lemma $2.3, \ell=1$ implies $G_{1}=[n]$ and $\operatorname{rank}(F)=$ $\operatorname{rank}([n])-1=r-1$. Since $[n] \backslash F=\sqcup_{k=1}^{\ell}\left(G_{k} \backslash F\right)$ by Lemma 2.3, we have

$$
\begin{aligned}
\ell\left(\sum_{j \in[n] \backslash F} a_{j}\right) & =\sum_{k=1}^{\ell}\left\{\left(\sum_{j \in[n] \backslash G_{k}} a_{j}\right)+\left(\sum_{j \in G_{k} \backslash F} a_{j}\right)\right\} \\
& =\sum_{k=1}^{\ell}\left(\sum_{j \in G_{k} \backslash F} a_{j}\right)=\sum_{j \in[n] \backslash F} a_{j},
\end{aligned}
$$

where we use the induction hypothesis to the second equality. As $\ell \geq 2$, the above equation implies (1) for $F$, as desired.

(ii) Let $f_{k}=\sum_{I \in \mathcal{I}(M),|I|=k}\left(\prod_{i \in I} x_{i}\right)$ for $k=0,1,2, \ldots, r$, where $f_{0}=1$. Then $P_{M}=x_{0}^{n}+x_{0}^{n-1} f_{1}+\cdots+x_{0}^{n-r} f_{r}$ and

$$
\bar{P}_{M}=\frac{n !}{r !} x_{0}^{r}+\frac{(n-1) !}{(r-1) !} x_{0}^{r-1} f_{1}+\frac{(n-2) !}{(r-2) !} x_{0}^{r-2} f_{2}+\cdots+(n-r) ! f_{r} .
$$

Suppose $\left(a_{0} \partial_{0}+a_{1} \partial_{1}+\cdots+a_{n} \partial_{n}\right) \bar{P}_{M}=0$ with $a_{0}, a_{1}, \ldots, a_{n} \in \mathbb{R}$. We will prove $a_{0}=a_{1}=\cdots=a_{n}=0$ or $M=U_{r, n}$. Since

$$
\begin{aligned}
& \left(a_{0} \partial_{0}+\cdots+a_{n} \partial_{n}\right) \bar{P}_{M} \\
& =\sum_{k=1}^{r} \frac{(n-k) !}{(r-k) !}\left\{(n-k+1) a_{0} f_{k-1}+\left(a_{1} \partial_{1}+\cdots+a_{n} \partial_{n}\right) f_{k}\right\} x_{0}^{r-k}
\end{aligned}
$$

equals to zero, we have

$$
(n-k+1) a_{0} f_{k-1}+\left(a_{1} \partial_{1}+\cdots+a_{n} \partial_{n}\right) f_{k}=0 \text { for } k=1,2, \ldots, r .
$$

Since $M$ is simple, $f_{1}=\sum_{k=1}^{n} x_{k}$ and $f_{2}=\sum_{1 \leq i<j \leq n} x_{i} x_{j}$, so by considering (2) when $k=2$ we have

$$
\sum_{k=1}^{n}\left\{(n-1) a_{0}+\sum_{j \neq k} a_{j}\right\} x_{k}=0 \Leftrightarrow \sum_{j \neq k} a_{j}=-(n-1) a_{0} \text { for } k=1,2, \ldots, n .
$$

This tells $a_{1}=a_{2}=\cdots=a_{n}=-a_{0}$ by Lemma 2.4 .

If $a_{0}=0$, then we have $a_{0}=\cdots=a_{n}=0$. Suppose $a_{0} \neq 0$. Then, by substituting $x_{1}=\cdots=x_{n}=1$ in $(2)$, we get

$$
a_{0}\left\{(n-k+1) I_{k-1}(M)-k I_{k}(M)\right\}=0
$$

for $k=1,2,3, \ldots, r$. This proves

$$
I_{k}(M)=\frac{n-k+1}{k} I_{k-1}(M)=\cdots=\frac{(n-k+1)(n-k+2) \cdots n}{k !}=\left(\begin{array}{l}
n \\
k
\end{array}\right)
$$

for $k=1,2, \ldots, r$, which tells $M=U_{r, n}$.

If $\partial_{0} f, \ldots, \partial_{n} f$ are $\mathbb{R}$-linearly dependent, then so do $\partial_{0}\left(\partial_{0} f\right), \ldots, \partial_{n}\left(\partial_{0} f\right)$. Thus the conclusion of Theorem 2.5(ii) also holds for $P_{M}$. Also, for a uniform matroid $U_{r, n}$, it is easy to see $\left(-\partial_{0}+\partial_{1}+\cdots+\partial_{n}\right) P_{U_{r, n}}=0$, so the statement (ii) does not hold for uniform matroids.

\section{SLP, HRR AND LORENTZIAN POLYNOMIALS}

In this section we discuss relations between the strong Lefschetz property, the Hodge-Riemann relation, and Lorentzian polynomials introduced by Brändén and Huh [BH2]. 
3.1. Lorentzian polynomials. A polynomial $f \in S$ is said to be log-concave (resp. strictly log-concave) on an open convex set $X \subset \mathbb{R}^{n}$ if the log of $f$ is a concave (resp. strictly concave) function on $X$. By a well-known criteria for the concavity, $\log f$ is concave on $X$ if and only if the Hessian matrix of $\log f$ is negative semidefinite at $\boldsymbol{x}=\boldsymbol{a}$ for any $\boldsymbol{a} \in X$, and $\log f$ is strictly concave on $X$ if the Hessian matrix of $\log f$ is negative definite at $\boldsymbol{x}=\boldsymbol{a}$ for any $\boldsymbol{a} \in X$. Note that when $f(\boldsymbol{a})>0$ the $\log$ of $f$ is negative semidefinite (resp. negative definite) at $\boldsymbol{x}=\boldsymbol{a}$ if and only if $\left.H_{f}\right|_{\boldsymbol{x}=\boldsymbol{a}}$ has exactly one positive eigenvalue (resp. has signature $(+,-, \ldots,-)$ ). See $[\mathrm{BH} 2$, Proposition 5.6] or [NY, §2.3]. We simply say that $f$ is log-concave at $\boldsymbol{a} \in \mathbb{R}^{n}$ if the Hessian matrix $\left.H_{f}\right|_{\boldsymbol{x}=\boldsymbol{a}}$ has exactly one positive eigenvalue.

Definition 3.1. Let $f \in \mathbb{R}_{\geq 0}\left[x_{1}, \ldots, x_{n}\right]$ be a homogeneous polynomial of degree $\geq 2$. We call that $f$ is a Lorentzian polynomial if for any $\left(k_{1}, \ldots, k_{n}\right) \in \mathbb{Z}_{\geq 0}^{n}$ with $\sum_{i=1}^{n} k_{i} \leq \operatorname{deg} f-2, \partial_{1}^{k_{1}} \cdots \partial_{n}^{k_{n}} f$ is identically zero or log-concave at any $\boldsymbol{a} \in \mathbb{R}_{>0}^{n}$.

The above property is also known as the strong log-concavity $[\mathrm{Gu}]$, but we call it Lorentzian since it is equivalent to the Lorentzian property defined in [BH2, Definition 2.1]. See [BH2, Theorem 5.3]. We note the next observation that follows from the continuity of eigenvalues.

Lemma 3.2. If $f \in \mathbb{R}_{\geq 0}\left[x_{1}, \ldots, x_{n}\right]$ is Lorentzian, then $\left.H_{f}\right|_{\boldsymbol{x}=\boldsymbol{a}}$ has at most one positive eigenvalue for any $\boldsymbol{a} \in \mathbb{R}_{\geq 0}^{n}$.

An important instance of Lorentzian polynomials are generating polynomials of matroids. Indeed, the following result is proved in [AOV, ALOVI, ALOVII, BH1, BH2] (see [AOV, Theorem 25] and [ALOVII, Theorem 4.1]).

Lemma 3.3. For any matroid $M$ of rank $\geq 2$, the polynomials $f_{M}$ and $P_{M}$ are Lorentzian.

3.2. The Strong Lefschetz property and the Hodge-Riemann relation. Lorentzian polynomials are related to algebraic properties called the strong Lefschetz property and the Hodge-Riemann relation.

Let $S=\mathbb{R}\left[\partial_{1}, \ldots, \partial_{n}\right]$ be the polynomial ring whose variables are $\partial_{1}, \ldots, \partial_{n}$. For a homogenous polynomial $f \in \mathbb{R}\left[x_{1}, \ldots, x_{n}\right]$ of degree $d$, we define the $\mathbb{R}$-algebra

$$
R_{f}^{*}:=S / \operatorname{Ann}_{S}(f),
$$

where $\operatorname{Ann}_{S}(f)=\{D \in S \mid D f=0\}$. It is well-known that $R_{f}^{*}$ is a Poincaré duality algebra, that is, $R_{f}^{d} \cong \mathbb{R}$ and the bilinear pairing induced by the multiplication $R_{f}^{k} \times R_{f}^{d-k} \rightarrow R_{f}^{d}$ is nondegenerate for all $k$ (see e.g. [MW, Theorem 2.1]). We say that $R_{f}^{*}$ (or $f$ ) has the strong Lefschetz property at degree $k \leq d / 2$ ( $\operatorname{shortly~} \mathrm{SLP}_{k}$ ) w.r.t. a linear form $\ell \in S$ if the multiplication map

$$
\times \ell^{d-2 k}: R_{f}^{k} \rightarrow R_{f}^{d-k}
$$

is an isomorphism. We say that $R_{f}^{*}$ (or $f$ ) satisfies the Hodge-Riemann relation at degree $k$ (shortly $\mathrm{HRR}_{k}$ ) w.r.t. a linear form $\ell \in S$ if $R_{f}^{*}$ has the $\mathrm{SLP}_{k}$ w.r.t. $\ell$ and the bilinear form

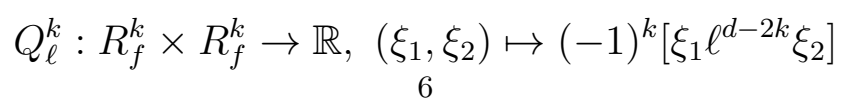


is positive definite on the kernel of $\times \ell^{d+1-2 k}: R_{f}^{k} \rightarrow R_{f}^{d-2 k+1}$, where $[-]: R_{f}^{d} \rightarrow \mathbb{R}$ is the isomorphism defined by $D \mapsto D\left(\partial_{1}, \ldots, \partial_{n}\right) f$.

We are actually only interested in $\mathrm{SLP}_{1}$ and $\mathrm{HRR}_{1}$ in this paper. For $\boldsymbol{a}=$ $\left(a_{1}, \ldots, a_{n}\right) \in \mathbb{R}^{n}$, we write $\ell_{\boldsymbol{a}}=a_{1} \partial_{1}+\cdots+a_{n} \partial_{n}$.

Lemma 3.4. Let $f \in S$ be a homogeneous polynomial of degree $\geq 2$ and $\boldsymbol{a} \in \mathbb{R}^{n}$. Assume that $f(\boldsymbol{a})>0$. Then,

(i) $R_{f}$ has the $\mathrm{SLP}_{1}$ w.r.t. $\ell_{\boldsymbol{a}} \Leftrightarrow Q_{\ell_{\boldsymbol{a}}}^{1}$ is non-singular.

(ii) $R_{f}$ has the $\mathrm{HRR}_{1}$ w.r.t. $\ell_{\boldsymbol{a}} \Leftrightarrow-Q_{\ell_{\boldsymbol{a}}}^{1}$ has signature $(+,-, \ldots,-)$.

Proof. The statement (i) is obvious. We show (ii). Define the map $\psi_{\boldsymbol{a}}: R_{f}^{1} \rightarrow R_{f}^{d}$ by $\psi_{\boldsymbol{a}}(h)=\ell_{\boldsymbol{a}}^{d-1} h$. Since the map

$$
\times \ell_{\boldsymbol{a}}^{d}: R_{f}^{0} \stackrel{\times \ell_{a}}{\longrightarrow} R_{f}^{1} \stackrel{\psi_{a}}{\longrightarrow} R_{f}^{d}
$$

is an isomorphism, the decomposition $R_{f}^{1}=\mathbb{R} \ell_{\boldsymbol{a}} \oplus \operatorname{Ker} \psi_{\boldsymbol{a}}$ is orthogonal with respect to $Q_{\ell_{\boldsymbol{a}}}^{1}$. Since $-Q_{\ell_{\boldsymbol{a}}}^{1}\left(\ell_{\boldsymbol{a}}, \ell_{\boldsymbol{a}}\right)=\left[\ell_{\boldsymbol{a}}^{d}\right]=d ! f(\boldsymbol{a})>0$, it follows that $R_{f}$ satisfies the $\mathrm{HRR}_{1}$ w.r.t. $\ell_{\boldsymbol{a}}$ if and only if $-Q_{\ell_{\boldsymbol{a}}}^{1}$ is nondegenerate and has only one positive eigenvalue.

The previous lemma implies the following fact.

Lemma 3.5. If $f \in \mathbb{R}_{\geq 0}\left[x_{1}, \ldots, x_{n}\right]$ is Lorentzian, then for any $\boldsymbol{a} \in R_{\geq 0}^{n}$ with $f(\boldsymbol{a})>0, f$ has the $\mathrm{SLP}_{1}$ w.r.t. $\ell_{\boldsymbol{a}}$ if and only if $f$ has the $\mathrm{HRR}_{1}$ w.r.t. $\ell_{\boldsymbol{a}}$.

Proof. The Hessian matrix $\left.H_{f}\right|_{\boldsymbol{x}=\boldsymbol{a}}$ is (a positive scalar multiple of) the representation of the symmetric bilinear form $-Q_{\ell_{a}}^{1}: R_{f}^{1} \times R_{f}^{1} \rightarrow \mathbb{R}$ w.r.t. the generating set $\left\{\partial_{1}, \ldots, \partial_{n}\right\}$ of $R_{f}^{1}$. Indeed, by definition, we have

$$
-Q_{\ell_{\boldsymbol{a}}}^{1}\left(\partial_{i}, \partial_{j}\right)=\left[\partial_{i} \ell_{\boldsymbol{a}}^{d-2} \partial_{j}\right]=\left(a_{1} \partial_{1}+\cdots+a_{n} \partial_{n}\right)^{d-2}\left(\partial_{i} \partial_{j} f\right)=\left.(d-2) !\left(\partial_{i} \partial_{j} f\right)\right|_{\boldsymbol{x}=\boldsymbol{a}},
$$

where $d=\operatorname{deg} f$. Since Sylvester's law tells that the number of positive eigenvalues of the symmetric matrix representing a fixed symmetric bilinear form does not depend on the choice of a generating set, the number of positive eigenvalues of $-Q_{\ell_{a}}$ equals to that of $\left.H_{f}\right|_{\boldsymbol{x}=\boldsymbol{a}}$. Then the assertion follows from Lemmas 3.2 and 3.4.

We also note the next fact, which immediately follows from the fact that $\partial_{1}, \ldots, \partial_{n}$ is an $\mathbb{R}$-basis of $R_{f}^{1}$ if and only if $\partial_{1} f, \ldots, \partial_{n} f$ are $\mathbb{R}$-linearly independent.

Lemma 3.6. Let $f \in \mathbb{R}\left[x_{1}, \ldots, x_{n}\right]$ be a homogeneous polynomial of degree $\geq 2$ and $\boldsymbol{a} \in \mathbb{R}^{n}$. If $\partial_{1} f, \ldots, \partial_{n} f$ are $\mathbb{R}$-linearly independent, then $R_{f}$ has the $\mathrm{SLP}_{1}$ (resp. $\mathrm{HRR}_{1}$ ) w.r.t. $\ell_{\boldsymbol{a}} \in S$ if and only if $\left.H_{f}\right|_{\boldsymbol{x}=\boldsymbol{a}}$ is non-singular (resp. has signature $(+,-, \ldots,-))$.

3.3. The local HRR and the SLP. We say that a homogeneous polynomial $f \in \mathbb{R}\left[x_{1}, \ldots, x_{n}\right]$ of degree $d \geq 2 k+1$ has the local $\mathrm{HRR}_{k}$ w.r.t. a linear form $\ell \in S$ if, for any $i=1,2, \ldots, n, \partial_{i} f$ is either zero or has the $\mathrm{HRR}_{k}$ w.r.t. $\ell$. The next proposition would be known for experts, but we include its proof since we cannot find a version which covers the case we need (see e.g., [AHK, Proposition 7.15] for a similar statement). 
Lemma 3.7. Let $f \in \mathbb{R}_{\geq 0}\left[x_{1}, \ldots, x_{n}\right]$ be a homogeneous polynomial of degree $d$ and $k$ a positive integer with $d \geq 2 k+1$, and $\boldsymbol{a}=\left(a_{1}, \ldots, a_{n}\right) \in \mathbb{R}^{n}$. Suppose that $f$ has the local $\mathrm{HRR}_{k}$ w.r.t. $\ell_{\boldsymbol{a}}$.

(i) If $\boldsymbol{a} \in \mathbb{R}_{>0}^{n}$, then $R_{f}$ has the $\mathrm{SLP}_{k}$ w.r.t. $\ell_{\boldsymbol{a}}$.

(ii) If $a_{1}=0, a_{2}, \ldots, a_{n}>0$ and $\left\{\xi \in R_{f}^{k} \mid \partial_{i} \xi=0\right.$ for $\left.i=2, \ldots, n\right\}=\{0\}$, then $R_{f}$ has the $\mathrm{SLP}_{k}$ w.r.t. $\ell_{\boldsymbol{a}}$.

Proof. We prove (i) and (ii) simultaneously. Without loss of generality, we may assume that $f \notin \mathbb{R}\left[x_{1}, \ldots, \hat{x}_{i}, \ldots, x_{n}\right]$ for any $i$. Consider the following two maps:

$$
\begin{gathered}
{[-]: R_{f}^{d} \stackrel{\sim}{\rightarrow} \mathbb{R}, h \mapsto[h]=h\left(\partial_{1}, \ldots, \partial_{n}\right) f,} \\
{[-]_{i}: R_{\partial_{i} f}^{d-1} \stackrel{\sim}{\rightarrow} \mathbb{R}, h^{\prime} \mapsto\left[h^{\prime}\right]_{i}=h^{\prime}\left(\partial_{1}, \ldots, \partial_{n}\right) \partial_{i} f .}
\end{gathered}
$$

Also, let $Q_{i}$ be the Hodge-Riemann bilinear form for $R_{\partial_{i} f}=S / \operatorname{Ann}_{S}\left(\partial_{i} f\right)$ with respect to $\ell_{a}$ :

$$
Q_{i}: R_{\partial_{i} f}^{k} \times R_{\partial_{i} f}^{k} \rightarrow \mathbb{R},(v, w) \mapsto Q_{i}(v, w)=(-1)^{k}\left[v \ell_{\boldsymbol{a}}^{d-2 k-1} w\right]_{i} .
$$

Suppose that $L \in R_{f}^{k}$ satisfies $L \ell_{\boldsymbol{a}}^{d-2 k}=0$ in $R_{f}^{d-k}$. To prove the desired statement, what we must prove is $L=0$ under the assumption of (i) or (ii). Since $L \ell_{\boldsymbol{a}}^{d-2 k}=0$ in $R_{\partial_{i} f}^{d-k}$ as well, $L \in R_{\partial_{i} f}^{k}$ is contained in the kernel of

$$
\times \ell_{\boldsymbol{a}}^{d-2 k}: R_{\partial_{i} f}^{k} \rightarrow R_{\partial_{i} f}^{d-k} .
$$

Since $Q_{i}$ is positive definite on the kernel of the above map, we have

$$
Q_{i}(L, L) \geq 0
$$

and $Q_{i}(L, L)=0$ if and only if $L=0$ in $R_{\partial_{i} f}=S / \operatorname{Ann}_{S}\left(\partial_{i} f\right)$. On the other hand, since $L \ell_{\boldsymbol{a}}^{d-2 k}=0$ in $R_{f}^{d-k}$, we have

$$
0=\left[L^{2} \ell_{\boldsymbol{a}}^{d-2 k}\right]=\left[\sum_{i=1}^{n} a_{i} \partial_{i} L^{2} \ell_{\boldsymbol{a}}^{d-2 k-1}\right]=\sum_{i=1}^{n} a_{i}\left[L^{2} \ell_{\boldsymbol{a}}^{d-2 k-1}\right]_{i}=(-1)^{k} \sum_{i=1}^{n} a_{i} Q_{i}(L, L) .
$$

Now assume $a_{i}>0$ for all $i$. We note that $\left\{\xi \in R_{f} \mid \partial_{i} \xi=0\right.$ for all $\left.i\right\}=R_{f}^{d}$ since, for any $D \in S$ of degree $<d$, if $D f \neq 0$ then $\partial_{i}(D f) \neq 0$ for some $i$. The above equation and (3) tell that $Q_{i}(L, L)=0$ for all $i$, and therefore $L=0$ in $R_{\partial_{i} f}$ for all $i$. But, since $\left\{\xi \in R_{f} \mid \partial_{i} \xi=0\right.$ for all $\left.i\right\}=R_{f}^{d}$, this implies $L=0$ in $R_{f}$, proving (i).

The proof of (ii) is similar. Indeed, if $a_{1}=0$ and $a_{2}, \ldots, a_{n}>0$, then the same argument tells $Q_{i}(L, L)=0$ and $L=0$ in $R_{\partial_{i} f}$ for all $i=2, \ldots, n$. Then $\partial_{i} L=0$ in $R_{f}$ for all $i=2, \ldots, n$, and the assumption of (ii) tells $L=0$ in $R_{f}$.

The following statement immediately follows from Lemmas 3.5 and 3.7, both of which are basic, but is crucial to prove Theorem 1.1(i).

Theorem 3.8. If $f \in \mathbb{R}\left[x_{1}, \ldots, x_{n}\right]$ is Lorentzian, then $f$ has the $\mathrm{HRR}_{1}$ w.r.t. $\ell_{\boldsymbol{a}}$ for any $\boldsymbol{a} \in \mathbb{R}_{>0}^{n}$.

Proof. By Lemma 3.5, we only have to show that $R_{f}$ has the $\mathrm{SLP}_{1}$ w.r.t. $\ell_{\boldsymbol{a}}$. We prove by induction on $d=\operatorname{deg} f$. When $d=2$, this is trivial since any degree 2 homogeneous polynomial has the $\mathrm{SLP}_{1}$ w.r.t. any linear form by definition. When $d \geq 3$, by Lemma 3.7, it suffices to show that for each $i$ with $\partial_{i} f \neq 0, \partial_{i} f$ satisfies the $\mathrm{HRR}_{1}$ w.r.t. $\ell_{\boldsymbol{a}}$. Since $\partial_{i} f$ is also a Lorentzian polynomial if it is non-zero 
by the definition of the Lorentzian property, the claim is trivial by the induction hypothesis.

Remark 3.9. Maeno-Numata $[\mathrm{MN}]$ conjectured that, for any matroid $M, R_{f_{M}}$ has the $\mathrm{SLP}_{k}$ for all $k$ w.r.t. some linear form $\ell$. Since $f_{M}$ is Lorentzian, the above statement verifies this conjecture when $k=1$.

\section{Proof of MAIN RESUlts}

In this section, we prove Theorem 1.1 and Corollary 1.2 in the introduction. We first prove Theorem 1.1. Since $f_{M}$ and $\bar{P}_{M}$ are Lorentzian, the statement (i) and the statement (ii) when $a_{0} \neq 0$ immediately follow from Theorems 2.5 and 3.8 together with Lemma 3.6. Then the next statement completes the proof of Theorem 1.1.

Theorem 4.1. Let $M$ be a matroid on $[n]$ of rank $r \geq 2$ and $\boldsymbol{a}=\left(a_{1}, \ldots, a_{n}\right) \in \mathbb{R}_{>0}^{n}$. Then $\bar{P}_{M}$ has the $\mathrm{HRR}_{1}$ w.r.t. $\ell_{\boldsymbol{a}}=a_{1} \partial_{1}+\cdots+a_{n} \partial_{n}$.

Proof. To prove this, we may assume that $M$ is loopless. Also, it suffices to prove that $\bar{P}_{M}$ has the $\mathrm{SLP}_{1}$ with respect to $\ell_{\boldsymbol{a}}$ since $\mathrm{SLP}_{1}$ and $\mathrm{HRR}_{1}$ are equivalent in this case by Lemma 3.5 . We prove that $\bar{P}_{M}$ has the $\mathrm{SLP}_{1}$ w.r.t. $\ell_{\boldsymbol{a}}$ by using induction on $r$.

If $M$ has rank 2, then the assertion is obvious because any degree 2 homogeneous polynomial has the $\mathrm{SLP}_{1}$.

Suppose that $M$ has rank $r \geq 3$. Since $\partial_{0} \bar{P}_{M}=\bar{P}_{T M}$ and $\partial_{i} \bar{P}_{M}=\bar{P}_{M / i}$, the induction hypothesis and Lemma 3.7(ii) tell that it suffices to prove

$$
\left\{L \in R_{\bar{P}_{M}}^{1} \mid \partial_{i} L=0 \text { for } i=1,2, \ldots, n\right\}=\{0\} .
$$

Note that $R_{\bar{P}_{M}}$ is a quotient ring of $\mathbb{R}\left[\partial_{0}, \ldots, \partial_{n}\right]$. Let $L=b_{0} \partial_{0}+b_{1} \partial_{1}+\cdots+b_{n} \partial_{n}$ and assume $\partial_{i} L \bar{P}_{M}=0$ for all $i=1,2, \ldots, n$. To prove (4), what we must prove is $L \bar{P}_{M}=0$.

Since $\partial_{i} L \bar{P}_{M}=0$ for all $i=1,2, \ldots, n$, we have

$$
L \bar{P}_{M}=c x_{0}^{r-1}
$$

for some $c \in \mathbb{R}$. Let $f_{k}=\sum_{I \in \mathcal{I}(M),|I|=k}\left(\prod_{i \in I} x_{i}\right)$ for $k=1, \ldots, r$. Then $\bar{P}_{M}=$ $\frac{n !}{r !} x_{0}^{r}+\frac{(n-1) !}{(r-1) !} x_{0}^{r-1} f_{1}+\frac{(n-2) !}{(r-2) !} x_{0}^{r-2} f_{2}+\cdots$, so $L \bar{P}_{M}$ is the polynomial of the form

$$
\frac{(n-1) !}{(r-1) !}\left(n b_{0}+\sum_{k=1}^{n} b_{k}\right) x_{0}^{r-1}+\frac{(n-2) !}{(r-2) !}\left((n-1) b_{0} f_{1}+\left(\sum_{k=1}^{n} b_{k} \partial_{k}\right) f_{2}\right) x_{0}^{r-2}+\cdots
$$

Since $f_{1}=\sum_{k=1}^{n} x_{k}$ and $f_{2}=\sum_{1 \leq i<j \leq n} x_{i} x_{j}$, comparing coefficients of $x_{0}^{r-1}$ and $x_{0}^{r-2}$ in $L \bar{P}_{M}=c x_{0}^{r-1}$, we have

$$
c=n b_{0}+b_{1}+\cdots+b_{n}
$$

and

$$
\sum_{k=1}^{n}\left((n-1) b_{0}+\sum_{j \neq k} b_{j}\right) x_{k}=0 .
$$

Then we have $\sum_{j \neq k} b_{j}=-(n-1) b_{0}$ for all $k=1,2, \ldots, n$, and therefore $b_{1}=$ $\cdots=b_{n}=-b_{0}$ by Lemma 2.4. This implies $c=n b_{0}+b_{1}+\cdots+b_{n}=0$, and $L \bar{P}_{M}=c x_{0}^{r-1}=0$ as desired. 
In the rest of this section, we prove Corollary 1.2. We need the following two technical lemmas.

Lemma 4.2. Let $f \in \mathbb{R}\left[x_{1}, \ldots, x_{n}\right]$ be a homogeneous polynomial having the $\mathrm{HRR}_{1}$ w.r.t. $\ell_{\boldsymbol{a}}$ with $\boldsymbol{a} \in \mathbb{R}^{n}$ and let $\ell_{1}, \ell_{2} \in S$ be linear forms. If $\ell_{1}$ and $\ell_{2}$ are $\mathbb{R}$-linearly independent in $\mathbb{R}_{f}^{1}$ and $\left(\ell_{1} \ell_{1} f\right)(\boldsymbol{a})>0$, then

$$
\operatorname{det}\left(\begin{array}{ll}
\left(\ell_{1} \ell_{1} f\right)(\boldsymbol{a}) & \left(\ell_{1} \ell_{2} f\right)(\boldsymbol{a}) \\
\left(\ell_{1} \ell_{2} f\right)(\boldsymbol{a}) & \left(\ell_{2} \ell_{2} f\right)(\boldsymbol{a})
\end{array}\right)<0
$$

Proof. Consider the bilinear form

$$
Q: R_{f}^{1} \times R_{f}^{1} \rightarrow \mathbb{R}, \quad\left(\xi_{1}, \xi_{2}\right) \mapsto \xi_{1} \xi_{2} \ell_{a}^{d-2} \cdot f,
$$

where $d=\operatorname{deg} f$. Observe that the subspace $W=\operatorname{span}_{\mathbb{R}}\left\{\ell_{1}, \ell_{2}\right\} \subset R_{f}^{1}$ has $\mathbb{R}$ dimension 2 by the assumption. Since $f$ has the $\mathrm{HRR}_{1}$ w.r.t. $\ell_{\boldsymbol{a}}$, the bilinear form $Q$ has signature $(+,-, \ldots,-)$, so the restriction of $Q$ to $W$ has signature $(+,-),(0,-)$, or $(-,-)$ by Cauchy's interlacing theorem (see [AOV, Lemma 2.4]). Since $Q\left(\ell_{1}, \ell_{1}\right)=(d-2) !\left(\ell_{1} \ell_{1} f\right)(\boldsymbol{a})>0$, the latter two cases cannot occur. Then, since the determinant in the statement is a representation matrix of the bilinear form $\left.Q\right|_{W}: W \times W \rightarrow \mathbb{R}$ (up to a positive scalar multiplication), it must be negative as $\left.Q\right|_{W}$ has signature $(+,-)$.

Lemma 4.3. Let $M$ be a matroid on $[n]$ of $\operatorname{rank} r \geq 2$ and $\boldsymbol{a}=\left(a_{1}, \ldots, a_{n}\right) \in R_{>0}^{n}$.

(i) Let $i, j \in[n]$ be non-loops and assume $\operatorname{dim}_{\mathbb{R}} R_{f_{M}}^{1} \geq 3$. If $i$ and $j$ are not parallel, then $\ell_{\boldsymbol{a}}, \partial_{i}, \partial_{j}$ are $\mathbb{R}$-linearly independent in $R_{f_{M}}$.

(ii) If $M \neq U_{r, n}$ then $\partial_{0}, \ell_{\boldsymbol{a}}$ are $\mathbb{R}$-linearly independent in $R_{\bar{P}_{M}}$.

Proof. By Theorem 2.5 and Lemma 2.2(iii), the $\mathbb{R}$-vector space $\left\{\ell_{\boldsymbol{a}} \in S \mid \ell_{a} f_{M}=0\right\}$ is generated by

$$
\left\{\partial_{k}-\partial_{k^{\prime}} \mid k \text { and } k^{\prime} \text { are parallel in } M\right\} \cup\left\{\partial_{k} \mid k \text { is a loop of } M\right\} \text {. }
$$

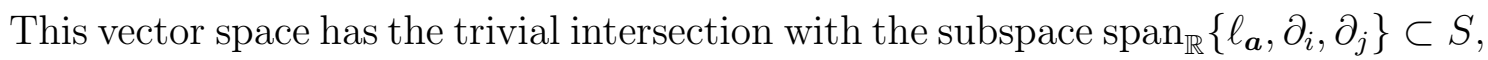
which guarantees (i). The proof for (ii) is similar.

Note that $\operatorname{dim}_{\mathbb{R}} R_{f_{M}}^{1}$ equals to the number of parallel classes of $M$,

We now prove Corollary 1.2. It is the special case of the following statement when $\boldsymbol{a}=(1,1, \ldots, 1)$.

Theorem 4.4. Let $M$ be a matroid on $[n]$ of rank $r \geq 2, i, j \in[n]$, and let $f_{k}=$ $\sum_{I \in \mathcal{I}(M),|I|=k}\left(\prod_{i \in I} x_{i}\right)$ for $k=0,1,2, \ldots, r$.

(i) If $i$ and $j$ are non-loops and $M$ has at least three parallel classes, then for any $\boldsymbol{a} \in \mathbb{R}_{>0}^{n}$ one has

$$
\left(f_{M}(\boldsymbol{a})\right) \times\left(\partial_{i} \partial_{j} f_{M}(\boldsymbol{a})\right)<2\left(1-\frac{1}{r}\right)\left(\partial_{i} f_{M}(\boldsymbol{a})\right) \times\left(\partial_{j} f_{M}(\boldsymbol{a})\right) .
$$

(ii) For any $\boldsymbol{a} \in \mathbb{R}_{>0}^{n}$ and $k+1 \geq \operatorname{girth}(M)$, one has

$$
\frac{f_{k-1}(\boldsymbol{a})}{\left(\begin{array}{c}
n \\
k-1
\end{array}\right)} \frac{f_{k+1}(\boldsymbol{a})}{\left(\begin{array}{c}
n \\
k+1
\end{array}\right)}<\left(\frac{f_{k}(\boldsymbol{a})}{\left(\begin{array}{l}
n \\
k
\end{array}\right)}\right)^{2} .
$$


We note that the non-strict inequalities are known and proved in [ALOVII, BH1, $\mathrm{BH} 2, \mathrm{HSW}$. In particular, our proof of the above theorem is based on the proofs of [BH1, Corollary 6] and [BH2, Lemma 4.4].

Proof. We first prove (i). If $i$ and $j$ are parallel in $M$, then $\partial_{i} \partial_{j} f_{M}=0$ so the assertion is obvious. We assume that $i$ and $j$ are not parallel.

To simplify the notation, we write

$$
f=f_{M}(\boldsymbol{a}), f_{i}=\left(\partial_{i} \cdot f_{M}\right)(\boldsymbol{a}), f_{j}=\left(\partial_{j} \cdot f_{M}\right)(\boldsymbol{a}) \text { and } f_{i j}=\left(\partial_{i} \partial_{j} \cdot f_{M}\right)(\boldsymbol{a}) .
$$

By Euler's identity, we have

$$
\left(\ell_{\boldsymbol{a}}^{2} \cdot f_{M}\right)(\boldsymbol{a})=r(r-1) f,\left(\ell_{a} \partial_{i} \cdot f_{M}\right)(\boldsymbol{a})=(r-1) f_{i} \text { and }\left(\ell_{a} \partial_{j} \cdot f_{M}\right)(\boldsymbol{a})=(r-1) f_{j} .
$$

Then, for any $t \in \mathbb{R}$, we have

$$
\begin{aligned}
& \frac{1}{r-1} \operatorname{det}\left(\begin{array}{cc}
\left(\ell_{\boldsymbol{a}}^{2} \cdot f_{M}\right)(\boldsymbol{a}) & \left(\ell_{\boldsymbol{a}}\left(\partial_{i}+t \partial_{j}\right) \cdot f_{M}\right)(\boldsymbol{a}) \\
\left(\ell_{\boldsymbol{a}}\left(\partial_{i}+t \partial_{j}\right) \cdot f_{M}\right)(\boldsymbol{a}) & \left(\left(\partial_{i}+t \partial_{j}\right)^{2} \cdot f_{M}\right)(\boldsymbol{a})
\end{array}\right) \\
& =\frac{1}{r-1} \operatorname{det}\left(\begin{array}{cc}
r(r-1) f & (r-1)\left(f_{i}+t f_{j}\right) \\
(r-1)\left(f_{i}+t f_{j}\right) & 2 t f_{i j}
\end{array}\right) \\
& =-(r-1) f_{j}^{2} t^{2}+2\left(r f f_{i j}-(r-1) f_{i} f_{j}\right) t-(r-1) f_{i}^{2} .
\end{aligned}
$$

The discriminant of the quadratic polynomial (5) in $t$ is

$$
\left(r f f_{i j}-(r-1) f_{i} f_{j}\right)^{2}-(r-1)^{2} f_{i}^{2} f_{j}^{2}=r f_{i} f_{j}\left(r f f_{i j}-2(r-1) f_{i} f_{j}\right) .
$$

Since $\ell_{\boldsymbol{a}}$ and $\partial_{i}+t \partial_{j}$ are $\mathbb{R}$-linearly independent in $R_{f_{M}}$ by Lemma 4.3 , the determinant in (5) is negative for all $t \in \mathbb{R}$ by Lemma 4.2. This tells that the discriminant (6) must be negative. Hence we have $r f f_{i j}<2(r-1) f_{i} f_{j}$, as desired.

(ii) Let $M^{\prime}=T^{r-k-1} M$. Since $k+1 \geq \operatorname{girth}(M), M^{\prime} \neq U_{r, n}$. Then, since

$$
\bar{P}_{M^{\prime}}=(n-k-1) ! f_{k+1}+(n-k) ! f_{k} \cdot x_{0}+\frac{(n-k+1) !}{2} f_{k-1} \cdot x_{0}^{2}+\cdots,
$$

we have

$$
\begin{aligned}
& \operatorname{det}\left(\begin{array}{cc}
\left(\ell_{\boldsymbol{a}}^{2} \bar{P}_{M^{\prime}}\right)\left(0, a_{1}, \ldots, a_{n}\right) & \left(\ell_{\boldsymbol{a}} \partial_{0} \cdot \bar{P}_{M^{\prime}}\right)\left(0, a_{1}, \ldots, a_{n}\right) \\
\left(\ell_{\boldsymbol{a}} \partial_{0} \cdot \bar{P}_{M^{\prime}}\right)\left(0, a_{1}, \ldots, a_{n}\right) & \left(\partial_{0}^{2} \cdot \bar{P}_{M^{\prime}}\right)\left(0, a_{1}, \ldots, a_{n}\right)
\end{array}\right) \\
& =\operatorname{det}\left(\begin{array}{cc}
(n-k-1) !\left(\ell_{\boldsymbol{a}}^{2} f_{k+1}\right)(\boldsymbol{a}) & (n-k) !\left(\ell_{\boldsymbol{a}} \cdot f_{k}\right)(\boldsymbol{a}) \\
(n-k) !\left(\ell_{\boldsymbol{a}} f_{k}\right)(\boldsymbol{a}) & (n-k+1) ! f_{k-1}(\boldsymbol{a})
\end{array}\right) \\
& =(n-k) !(n-k-1) ! \operatorname{det}\left(\begin{array}{cc}
(k+1) k f_{k+1}(\boldsymbol{a}) & (n-k) k f_{k}(\boldsymbol{a}) \\
(n-k) k f_{k}(\boldsymbol{a})(\boldsymbol{a}) & (n-k+1) f_{k-1}(\boldsymbol{a})
\end{array}\right) \\
& =k(n-k) !(n-k-1) !\left\{(n-k+1)(k+1) f_{k+1}(\boldsymbol{a}) f_{k-1}(\boldsymbol{a})-(n-k) k\left(f_{k}(\boldsymbol{a})\right)^{2}\right\} .
\end{aligned}
$$

Recall that $\bar{P}_{M^{\prime}}$ has the $\mathrm{HRR}_{1}$ w.r.t. $\ell_{\boldsymbol{a}}=a_{1} \partial_{1}+\cdots+a_{n} \partial_{n}$ by Theorem 4.1 . Then the above determinant must be negative by Lemmas 4.2 and 4.3 . Hence we have

$$
(n-k+1)(k+1) f_{k+1}(\boldsymbol{a}) f_{k-1}(\boldsymbol{a})-(n-k) k\left(f_{k}(\boldsymbol{a})\right)^{2}<0 .
$$

It is easy to see that this inequality is the same as the desired inequality. 


\section{MORPHISM OF MATROIDS}

Recently, Eur-Huh [EH] extend the Lorentzian property of $f_{M}$ and $P_{M}$ to basis generating polynomials of morphisms of matroids. In this section, we generalize Theorem 2.5 to morphisms of matroids. Note that, by Theorem 3.8, this partially generalize Theorem 1.1.

Definition 5.1. Let $M$ be a matroid on $[n]$ of rank $r$ and $N$ a matroid of rank $r^{\prime}$. A morphism $\varphi: M \rightarrow N$ is a map between the underlying space satisfying the following equivalent conditions:

(i) For any $S_{1} \subset S_{2} \subset[n]$, we have

$$
\operatorname{rank}_{N}\left(\varphi\left(S_{2}\right)\right)-\operatorname{rank}_{N}\left(\varphi\left(S_{1}\right)\right) \leq \operatorname{rank}_{M} S_{2}-\operatorname{rank}_{M} S_{1}
$$

(ii) For any flat $F$ of $N, \varphi^{-1}(F)$ is a flat of $M$.

We refer the readers to $[\mathrm{EH}]$ for basic properties and typical instances of morphisms of matroids.

Let $\varphi: M \rightarrow N$ be as in Definition 5.1. A subset $I \subset[n]$ is a basis of $\varphi$ if $I$ is an independent set of $M$ and $\langle\varphi(I)\rangle$ equals to the ground set of $N$, equivalently, $\operatorname{rank}(\varphi(I))=\operatorname{rank} N$. We write $\mathcal{B}(\varphi)$ for the set of bases of $\varphi$. Also, for $k \geq 0$, we write $\mathcal{B}(\varphi)_{k}=\{I \in \mathcal{B}(\varphi)|| I \mid=k\}$. We define the basis generating polynomial $P_{\varphi}$ of $\varphi$ as

$$
P_{\varphi}:=\sum_{I \in \mathcal{B}(\varphi)} x_{0}^{n-|I|}\left(\prod_{i \in I} x_{i}\right) .
$$

Also, we call

$$
\bar{P}_{\varphi}=\partial_{0}^{n-r} P_{\varphi}
$$

the reduced basis generating polynomial of $\varphi$, where $r=\operatorname{rank}(M)$. Below we give a few remarks on $\mathcal{B}(\varphi)$ and $P_{\varphi}$.

Remark 5.2. Let $\varphi$ be as above.

- $P_{\varphi}$ is non-trivial only when $\varphi([n])$ has rank $r^{\prime}$ in $N$. We assume this throughout the paper.

- $\mathcal{B}(\varphi)=\bigsqcup_{k=r^{\prime}}^{r} \mathcal{B}(\varphi)_{k}$ and $\mathcal{B}(\varphi)_{r}=\mathcal{B}(M)$. Also, $\left([n], \mathcal{B}(\varphi)_{k}\right)$ is a matroid for any $r^{\prime} \leq k \leq r$ (see the remark at the end of [EH, section 2]).

- When $r=r^{\prime}$, then $P_{\varphi}=x_{0}^{n-r} f_{M}$ and $\bar{P}_{\varphi}=(n-r) ! f_{M}$. Also, if $N=U_{0,1}$, then we have $P_{\varphi}=P_{M}$ and $\bar{P}_{\varphi}=\bar{P}_{M}$. From this viewpoint, basis generating polynomials of morphisms can be seen as a generalization of basis generating polynomials and independent set generating polynomials.

Let $M$ be a matroid on $[n]$. For any morphism $\varphi: M \rightarrow N$, we say that two elements $i$ and $j$ in $[n]$ are $\varphi$-parallel if $\varphi(i)$ and $\varphi(j)$ are parallel in $N$. We define $\varphi$-parallel classes in the same way as usual parallel classes. Also $i \in[n]$ is said to be a $\varphi$-loop if $\varphi(i)$ is a loop of $N$. We set $L_{\varphi}:=\{i \in[n] \mid i$ is a $\varphi$-loop $\}$.

By [EH, Corollary 22], $P_{\varphi}$ is a Lorentzian polynomial. Thus its Hessian matrix has signature $(+,-, \cdots-)$ when $\partial_{0} \bar{P}_{\varphi}, \partial_{1} \bar{P}_{\varphi}, \ldots, \partial_{n} \bar{P}_{\varphi}$ are $\mathbb{R}$-linearly independent. As the next example shows, this linear independency does not hold for all morphisms.

Example 5.3. Let $\varphi: M \rightarrow N$ be as in Definition 5.1. 
(1) If $i$ is a loop of $M$, then $\partial_{i} P_{\varphi}=0$. Similarly, if $i$ and $j$ are parallel in $M$, then $\left(\partial_{i}-\partial_{j}\right) P_{\varphi}=0$.

(2) If $r=r^{\prime}$, then $P_{\varphi}=f_{M}$. In this case, $\partial_{0} \bar{P}_{\varphi}=0$ since $\bar{P}_{\varphi}=(n-r) ! f_{M}$ does not contain $x_{0}$.

(3) Suppose that $r-r^{\prime}=1$ and $L_{\varphi}=\{1\}$. Then it is not hard to see

$$
P_{\varphi}=x_{0}^{n-r}\left(x_{0}+x_{1}\right) \sum_{I \in \mathcal{B}(\varphi)_{r^{\prime}}}\left(\prod_{i \in I} x_{i}\right)+x_{0}^{n-r} \sum_{1 \notin I \in \mathcal{B}(\varphi)_{r}}\left(\prod_{i \in I} x_{i}\right) .
$$

In this case, $\left(\partial_{0}-(n-r+1) \partial_{1}\right) \bar{P}_{\varphi}=0$.

(4) Suppose that $\left.M\right|_{L_{\varphi}}$ is a uniform matroid on $L_{\varphi}$ and $\left|[n] \backslash L_{\varphi}\right|=r^{\prime}$. Then it is not difficult to see

$$
P_{\varphi}=P_{\left.M\right|_{L \varphi}} \times\left(\prod_{i \in[n] \backslash L_{\varphi}} x_{i}\right) .
$$

(See also Lemma 5.4 below). In this case, $\left(-\partial_{0}+\sum_{i \in L_{\varphi}} \partial_{i}\right) P_{\varphi}=0$.

Here is an instance of such a morphism. Consider the morphism $\varphi$ : $U_{r-r^{\prime}, n-r^{\prime}} \bigoplus U_{r^{\prime}, r^{\prime}} \rightarrow N=U_{0,1} \bigoplus U_{r^{\prime}, r^{\prime}}$ which send elements in $U_{r, n}$ to the loop of $N$ (i.e. the element of $U_{0,1}$ ) and whose restriction to $U_{r^{\prime}, r^{\prime}}$ is an isomorphism. This map is indeed a morphism of matroids and satisfies the above condition.

We will prove that these are the only cases that the linear dependency of the polynomial $\partial_{0} \bar{P}_{\varphi}, \ldots, \partial_{n} \bar{P}_{\varphi}$ occurs. For the proof, we need the following lemmas.

Lemma 5.4. Let $\varphi: M \rightarrow N$ be a morphism of matroids and $I \in \mathcal{B}(\varphi) \operatorname{rank}(N)$. Then $I \cap L_{\varphi}=\emptyset$ and, for any $J \subset L_{\varphi}$, one has $I \cup J \in \mathcal{B}(\varphi)$ if and only if $J \in \mathcal{I}\left(\left.M\right|_{L_{\varphi}}\right)$.

Proof. Let $I \in \mathcal{B}(\varphi)_{\operatorname{rank}(N)}$. If $I$ contains a $\varphi$-loop $j$, then $\operatorname{rank}(N)=\operatorname{rank}(\varphi(I))=$ $\operatorname{rank}(\varphi(I \backslash\{j\}))$, so $\operatorname{rank}(N) \leq|I \backslash\{j\}|<|I|$, contradicting $|I|=\operatorname{rank}(N)$. Also, for any $J \subset L_{\varphi}$, since

$$
\operatorname{rank}(I \cup J)-\operatorname{rank}(J) \geq \operatorname{rank}(\varphi(I \cup J))-\operatorname{rank}(\varphi(J))=|I|-0,
$$

one has $I \cup J \in \mathcal{I}(M)$ if and only if $\operatorname{rank}(J)=|J|$. The first condition is equivalent to $I \cup J \in \mathcal{B}(\varphi)$ since $\operatorname{rank}(\varphi(I \cup J))=\operatorname{rank}(\varphi(I))=\operatorname{rank}(N)$, and the latter condition is equivalent to $J \in \mathcal{I}\left(\left.M\right|_{L_{\varphi}}\right)$.

Lemma 5.5. Let $m$ be a positive integer, $M$ a simple matroid on $[n]$ of rank $r \geq 2$, and $f=\partial^{n-r+m}\left(x_{0}^{m} P_{M}\right)$. Then $\partial_{0} f, \partial_{1} f, \ldots, \partial_{n} f$ are $\mathbb{R}$-linearly independent.

Proof. Let $\ell=\sum_{k=0}^{n} a_{k} \partial_{k}$ with $a_{k} \in \mathbb{R}$. Then $\ell f$ is a polynomial of the form

$$
c_{0}\left\{(n+m) a_{0}+\sum_{k=1}^{n} a_{k}\right\} x_{0}^{r-1}+c_{1}\left\{\sum_{k=1}^{n}\left((n+m-1) a_{0}+\sum_{j \neq k} a_{j}\right) x_{k}\right\} x_{0}^{r-2}+\cdots,
$$

where $c_{0}=\frac{(n+m-1) !}{(r-1) !}$ and $c_{1}=\frac{(n+m-2) !}{(r-2) !}$. Suppose $\ell f=0$. Then we have (i) $(n+$ m) $a_{0}+\sum_{k=1}^{n} a_{k}=0$ and (ii) $(n+m-1) a_{0}+\sum_{j \neq k} a_{j}=0$ for $k=1,2, \ldots, n$. The condition (ii) tells $a_{j}=-\frac{n+m-1}{n-1} a_{0}$ for all $j$ by Lemma 2.4, but then condition (i) says $0=(n+m) a_{0}-\frac{n(n+m-1)}{n-1} a_{0}=-\frac{m}{n-1} a_{0}$. Then we have $a_{0}=\cdots=a_{n}=0$, so $\partial_{0} f, \partial_{1} f, \ldots, \partial_{n} f$ are linearly independent. 
Now we prove the main result of this section.

Theorem 5.6. Let $M$ be a simple matroid on $[n]$ of rank $r, N$ a matroid of rank $r^{\prime}$, and $\varphi: M \rightarrow N$ a morphism of matroids such that $\operatorname{rank}_{N}(\varphi([n]))=r^{\prime}$. Then $\partial_{0} \bar{P}_{\varphi}, \partial_{1} \bar{P}_{\varphi}, \ldots, \partial_{n} \bar{P}_{\varphi}$ are $\mathbb{R}$-linearly dependent if and only if one of the following holds:

(A) $r=r^{\prime}$;

(B) $r-r^{\prime}=1$ and $\left|L_{\varphi}\right|=1$;

(C) $\left.M\right|_{L_{\varphi}}$ is a uniform matroid and $\left|[n] \backslash L_{\varphi}\right|=r^{\prime}$.

Proof. Let $\ell=\sum_{k=0}^{n} a_{k} \partial_{k}$ be non-zero, where $a_{0}, \ldots, a_{n} \in \mathbb{R}$, and assume $\ell \bar{P}_{\varphi}=0$. We prove that $\varphi$ satisfies one of $(\mathrm{A}),(\mathrm{B})$ and $(\mathrm{C})$. To prove this, we may assume $r>r^{\prime}$. Recall that

$$
\bar{P}_{\varphi}=\sum_{I \in \mathcal{B}(\varphi)} \frac{(n-|I|) !}{(r-|I|) !} x_{0}^{r-|I|}\left(\prod_{i \in I} x_{i}\right) .
$$

We first prove the next claim.

\section{Claim 1.}

(I) $a_{0} \neq 0$.

(II) If $E \subset[n]$ is a $\varphi$-parallel class, then $\sum_{i \in E} a_{i}=0$.

(III) For any flat $F$ of $M$ such that $\operatorname{rank}(\varphi(F))=\operatorname{rank}(F)$, one has $\sum_{[n] \backslash F} a_{i}=$ $-\left(n-r^{\prime}\right) a_{0}$.

(IV) For any $j \in[n] \backslash L_{\varphi}$, we have $a_{j}=0$.

Proof of Claim. (I) This follows from Theorem 2.5(i) since $\bar{P}_{\varphi}=(n-r)$ ! $f_{M}+x_{0} g$ for some polynomial $g \neq 0$.

(II) Recall that $M^{\prime}=\left([n], \mathcal{B}(\varphi)_{r^{\prime}}\right)$ is a matroid on $[n]$. Clearly $X \in \mathcal{B}(\varphi)_{r^{\prime}}$ if and only if $\operatorname{rank}(\varphi(X))=r^{\prime}$ for any $X \subset[n]$. From this fact, it is easy to see that, for any $X \subset[n]$, the rank of $X$ in $M^{\prime}$ equals to the rank of $\varphi(X)$ in $N$. In particular, $i, j \in[n]$ are parallel in $M^{\prime}$ if and only if they are $\varphi$-parallel. Since $P_{\varphi}$ can be written in the form $P_{\varphi}=x_{0}^{n-r^{\prime}} f_{M^{\prime}}+h$, where $h$ is a polynomial that contains no monomial divisible by $x_{0}^{n-r^{\prime}}, \ell \bar{P}_{\varphi}$ can be written as

$$
\ell \bar{P}_{\varphi}=\frac{\left(n-r^{\prime}\right) !}{\left(r-r^{\prime}\right) !} x_{0}^{r-r^{\prime}}\left(a_{1} \partial_{1}+\cdots+a_{n} \partial_{n}\right) f_{M^{\prime}}+h^{\prime}
$$

for some polynomial $h^{\prime}$ containing no monomials divisible by $x_{0}^{r-r^{\prime}}$. Since $\ell \bar{P}_{\varphi}=0$, we have $\left(a_{1} \partial_{1}+\cdots+a_{n} \partial_{n}\right) f_{M^{\prime}}=0$. Then by Lemma 2.2(iii) and Theorem 2.5(i) it follows that $a_{1} \partial_{1}+\cdots+a_{n} \partial_{n}$ belongs to

$$
\operatorname{span}_{\mathbb{R}}\left\{\left\{\partial_{i} \mid i \text { is an } \varphi \text {-loop }\right\} \cup\left\{\partial_{i}-\partial_{j} \mid i \text { and } j \text { are } \varphi \text {-parallel }\right\}\right\} .
$$

This guarantees the desired property.

(III) The proof is similar to that of Theorem 2.5(i). Suppose that $F$ has rank $r^{\prime}$. Let $I$ be an independent set of $M$ such that $\langle I\rangle=F$. Note that $|I|=\operatorname{rank}(F)=r^{\prime}$. 
A routine computation tells that the coefficient of $x_{0}^{r-r^{\prime}-1} \prod_{i \in I} x_{i}$ in $\ell \bar{P}_{\varphi}$ is

$$
\begin{aligned}
& \frac{\left(n-r^{\prime}\right) !}{\left(r-r^{\prime}\right) !}\left(r-r^{\prime}\right) a_{0}+\frac{\left(n-r^{\prime}-1\right) !}{\left(r-r^{\prime}-1\right) !}\left(\sum_{\{j\} \cup I \in \mathcal{I}(M), j \notin I} a_{j}\right) \\
& =\frac{\left(n-r^{\prime}-1\right) !}{\left(r-r^{\prime}-1\right) !}\left\{\left(n-r^{\prime}\right) a_{0}+\sum_{j \in[n] \backslash F} a_{j}\right\}
\end{aligned}
$$

(see also $(7))$. Since $\ell \bar{P}_{\varphi}=0$, this proves the desired equation for $F$.

Now suppose that $F$ has rank $<r^{\prime}$ and (III) holds for all flats $G \supsetneq F$ of $M$ with $\operatorname{rank}(\varphi(G))=\operatorname{rank}(G)$. If $G$ is a smallest flat of $M$ that properly contains $F$, then

$$
\operatorname{rank}(\varphi(G))-\operatorname{rank}(\varphi(F)) \leq \operatorname{rank} G-\operatorname{rank} F=1,
$$

so the rank of $\varphi(G)$ must be either $\operatorname{rank}(F)+1$ or $\operatorname{rank}(F)$. Let $G_{1}, \ldots, G_{p}, G_{1}^{\prime}, \ldots, G_{q}^{\prime}$ be the minimal flats of $M$ that property contain $F$, where $\operatorname{rank}\left(\varphi\left(G_{k}\right)\right)=\operatorname{rank}(F)+1$ and $\operatorname{rank}\left(\varphi\left(G_{k}^{\prime}\right)\right)=\operatorname{rank}(F)$. By Lemma 2.3, we have

$$
[n] \backslash F=\bigsqcup_{t=1}^{p}\left(G_{t} \backslash F\right) \sqcup \bigsqcup_{s=1}^{q}\left(G_{s}^{\prime} \backslash F\right) .
$$

We claim

Claim 2. $\bigsqcup_{t=1}^{p}\left(G_{t} \backslash F\right)$ is non-empty and a union of $\varphi$-parallel classes.

Proof of Claim 2. Note that the definition of $G_{1}, \ldots, G_{p}$ says that $k \in \bigsqcup_{t=1}^{p}\left(G_{t} \backslash F\right)$ if and only if $\operatorname{rank}(\varphi(\{k\} \cup F))=\operatorname{rank}(F)+1$. This in particular tells that $\bigsqcup_{t=1}^{p}\left(G_{t} \backslash F\right)$ is non-empty and contains no $\varphi$-loops. If $i$ and $j$ are $\varphi$-parallel and $i \in \bigsqcup_{t=1}^{p=1}\left(G_{t} \backslash F\right)$ then we have

$$
\operatorname{rank}(\varphi(\{j\} \cup F))=\operatorname{rank}(\varphi(\{i\} \cup F))=\operatorname{rank}(\varphi(F))+1,
$$

which tells that $j \in \sqcup_{t=1}^{p}\left(G_{t} \backslash F\right)$. This guarantees the desired property.

Now, by statement (II), we have $\sum_{j \in \bigsqcup_{t=1}^{p}\left(G_{t} \backslash F\right)} a_{j}=0$. Then

$$
\begin{aligned}
p \cdot\left(\sum_{j \in[n] \backslash F} a_{j}\right) & =\sum_{k=1}^{p}\left\{\sum_{j \in[n] \backslash G_{k}} a_{j}+\sum_{j \in G_{k} \backslash F} a_{j}\right\} \\
& =\sum_{k=1}^{p}\left(\sum_{j \in[n] \backslash G_{k}} a_{j}\right) \\
& =p \times\left(n-r^{\prime}\right) a_{0},
\end{aligned}
$$

which proves the desired property, where we use the induction hypothesis to the third equality.

(IV) If $\left|[n] \backslash L_{\varphi}\right| \leq 1$, then the assertion follows from the statement (II). We assume $\left|[n] \backslash L_{\varphi}\right| \geq 2$. Let $\alpha=\left(n-r^{\prime}\right) a_{0}+\sum_{i \in L_{\varphi}} a_{j}$. The statement (III) for rank 1 flats tells that for any $k \in[n] \backslash L_{\varphi}$, we have $\sum_{j \neq k} a_{j}=-\left(n-r^{\prime}\right) a_{0}$, equivalently, $\sum_{j \in[n] \backslash L_{\varphi}, j \neq k} a_{j}=-\alpha$. Then Lemma 2.4 tells $a_{j}=-\frac{1}{\left|[n] \backslash L_{\varphi}\right|-1} \alpha$ for all $j \in[n] \backslash L_{\varphi}$. 
Moreover, (II) tells, for any $j \in[n] \backslash L_{\varphi}$, we have $0=\sum_{i \text { is } \varphi \text {-parallel to } j} a_{i}=c \alpha$ for some $c<0$, so $\alpha=0$. These prove the desired statement.

We now go back to the proof of Theorem 5.6. By Claim 1, we have

$$
\ell=a_{0} \partial_{0}+\sum_{i \in L_{\varphi}} a_{i} \partial_{i}
$$

For each $I \in \mathcal{B}(\varphi)$ with $I \subset[n] \backslash L_{\varphi}$, let

$$
N_{I}=\left\{J \subset L_{\varphi} \mid J \cup I \in \mathcal{B}(\varphi)\right\} .
$$

Note that $N_{I}$ is the set of independent sets of the simple matroid obtained from $M$ by contracting elements in $I$ and then restrict it to $L_{\varphi}$. Also,

$$
P_{\varphi}=\sum_{I \in \mathcal{B}(\varphi), I \subset[n] \backslash L_{\varphi}} x_{0}^{n-|I|-\left|L_{\varphi}\right|} \cdot P_{N_{I}} \cdot\left(\prod_{i \in I} x_{i}\right) .
$$

Then, since

$$
\ell \bar{P}_{\varphi}=\ell \partial_{0}^{n-r} P_{\varphi}=\sum_{I \in \mathcal{B}(\varphi), I \subset[n] \backslash L_{\varphi}}\left\{\ell \partial_{0}^{n-r} \cdot\left(x_{0}^{n-|I|-\left|L_{\varphi}\right|} P_{N_{I}}\right)\right\}\left(\prod_{i \in I} x_{i}\right),
$$

we have

$$
\ell \partial_{0}^{n-r}\left(x_{0}^{n-|I|-\left|L_{\varphi}\right|} P_{N_{I}}\right)=0
$$

for all $I \in \mathcal{B}(\varphi)$ with $I \subset[n] \backslash L_{\varphi}$. Also, by Lemma 5.4, $N_{I}=\left.M\right|_{L_{\varphi}}$ for all $I \in \mathcal{B}(\varphi)$ with $|I|=r^{\prime}$. Then by Theorem 2.5(ii) and Lemma 5.5, we have either

(a) $\operatorname{rank}\left(\left.M\right|_{L_{\varphi}}\right) \leq 1$ or $\left.(\boldsymbol{(}) \quad M\right|_{L_{\varphi}}$ is a uniform matroid and $n-r^{\prime}-\left|L_{\varphi}\right|=0$.

The latter case is nothing but the condition (C). Suppose $\operatorname{rank}\left(\left.M\right|_{L_{\varphi}}\right) \leq 1$. Then $L_{\varphi}=\emptyset$ or $\left|L_{\varphi}\right|=1$. The former case cannot occur since $L_{\varphi}=\emptyset$ implies $\ell=a_{0} \partial_{0}$ and the assumption $r>r^{\prime}$ tells that $\bar{P}_{\varphi}$ contains a monomial divisible by $x_{0}$. Suppose $L_{\varphi}=\left\{j_{0}\right\}$ for some $j_{0} \in[n]$. Then $\ell=a_{0} \partial_{0}+a_{j_{0}} \partial_{j_{0}}$. Since $a_{0} \neq 0$ and $a_{0} \partial_{0} \bar{P}_{\varphi}=$ $-a_{j_{0}} \partial_{j_{0}} \bar{P}_{\varphi}+\ell \bar{P}_{\varphi}=-a_{j_{0}} \partial_{j_{0}} \bar{P}_{\varphi}$, we have

$$
a_{0}^{2} \partial_{0}^{2} \bar{P}_{\varphi}=a_{j_{0}}^{2} \partial_{j_{0}}^{2} \bar{P}_{\varphi}=0 .
$$

(Recall that $\bar{P}_{\varphi}$ contains no monomials which is divisible by $x_{k}^{2}$ for any $k \in[n]$.) This tells that $\bar{P}_{\varphi}$ contains no monomial which is divisible by $x_{0}^{2}$. This happens only when $r-r^{\prime} \leq 1$. Hence we have $\left|L_{\varphi}\right|=1$ and $r-r^{\prime}=1$, so condition (B) is satisfied.

Using the Lorentzian property of $P_{\varphi}$, Eur-Huh $[\mathrm{EH}]$ proved

$$
\frac{\left|\mathcal{B}(\varphi)_{k-1}\right|}{\left(\begin{array}{c}
n \\
k-1
\end{array}\right)} \frac{\left|\mathcal{B}(\varphi)_{k+1}\right|}{\left(\begin{array}{c}
n \\
k+1
\end{array}\right)} \leq\left(\frac{\left|\mathcal{B}(\varphi)_{k}\right|}{\left(\begin{array}{l}
n \\
k
\end{array}\right)}\right)^{2} \quad\left(r^{\prime}<k<r\right) .
$$

Considering Corollary 1.2, it is natural to ask

Question 5.7. When equality holds in the above inequality?

In the proof of Corollary 1.2, we use the property that $\bar{P}_{M}$ has the $\mathrm{HRR}_{1}$ w.r.t. $\partial_{1}+\cdots+\partial_{n}$. We close this paper with an example showing that this is not the case for morphisms of matroids. 
Example 5.8. Let $\varphi: U_{3,3} \rightarrow U_{1,1}$ be a (unique) natural morphism. Then,

$$
\bar{P}_{\varphi}=x_{1} x_{2} x_{3}+x_{0}\left(x_{1} x_{2}+x_{1} x_{3}+x_{2} x_{3}\right)+x_{0}^{2}\left(x_{1}+x_{2}+x_{3}\right)
$$

and a routine computation tells that $\bar{P}_{\varphi}$ does not have the $\mathrm{SLP}_{1}$ w.r.t. $\partial_{1}+\partial_{2}+\partial_{3}$.

\section{REFERENCES}

[AOV] N. Anari, S. Oveis Gharan, and C. Vinzant, Log-concave polynomials, entropy, and a deterministic approximation algorithm for counting bases of matroids, 59th Annual IEEE Symposium on Foundations of Computer Science-FOCS 2018, 35-46, IEEE Computer Soc., Los Alamitos, CA, 2018.

[ALOVI] N. Anari, K. Liu, S. Oveis Gharan, and C. Vinzant, Log-Concave Polynomials II: HighDimensional Walks and an FPRAS for Counting Bases of a Matroid, arXiv:1811.01816.

[ALOVII] N. Anari, K. Liu, S. Oveis Gharan, and C. Vinzant, Log-Concave Polynomials III: Mason's Ultra-Log-Concavity Conjecture for Independent Sets of Matroids, arXiv:1811.01600.

[AHK] K. Adiprasito, J. Huh, and E. Katz, Hodge theory for combinatorial geometries, Ann. of Math. (2) 188(2018), 381-452.

[BH1] P. Brändén and J. Huh, Hodge-Riemann relations for Potts model partition functions, arXiv: 1811.01696.

[BH2] P. Brändén and J. Huh, Lorentzian polynomials, arXiv:1902.03719.

[COSW] Y. Choe, J. Oxley, A. Sokal, and D. Wagner, Homogeneous multivariate polynomials with the half-plane property, Adv. in Appl. Math. 32 (2004), no. 1-2, 88-187.

[EH] C. Eur and J. Huh, Logarithmic concavity for morphisms of matroids, Adv. Math., to appear, arXiv:1906.00481.

[Gu] L. Gurvits, On multivariate Newton-like inequalities, Advances in combinatorial mathematics, 61-78, Springer, Berlin, 2009.

[HSW] J. Huh, B. Schröter, and B. Wang, Correlation bounds for fields and matroids, arXiv:1806.02675.

[HW] J. Huh and B. Wang, Enumeration of points, lines, planes, etc, Acta Math. 218 (2017), pp. $297-317$.

[MN] T. Maeno and Y. Numata, Sperner property and finite-dimensional Gorenstein algebras associated to matroids, Journal of Commutative Algebra 8 (2016), no.4, 549-570.

[MW] T. Maeno and J. Watanabe, Lefschetz elements of Artinian Gorenstein algebras and Hessians of homogeneous polynomials, Illinois J. Math. 53 (2009), no. 2, 591-603.

[NY] T. Nagaoka and A. Yazawa, Strictly log-concavity of the Kirchhoff polynomial and its applications to the strong Lefschetz property, arXiv:1904.01800.

[Ox] J. Oxley, Matroid theory, Second edition, Oxford Graduate Texts in Mathematics, vol. 21, Oxford University Press, Oxford, 2011.

[Ya] A. Yazawa, The Hessians of the complete and complete bipartite graphs and its application to the strong Lefschetz property, arXiv:1812.07199.

(Satoshi Murai) Department of Mathematics Faculty of Education Waseda UniverSity, 1-6-1 Nishi-Waseda, SHINJuku, TOKYo 169-8050, JAPAN

E-mail address: s-murai@waseda.jp

(Takahiro Nagaoka) Department of Mathematics, Graduate School of Science, KyOTO UNIVERSITY, KYOTO, 606-8522, JAPAN

E-mail address: tnagaoka@math.kyoto-u.ac.jp

(Akiko Yazawa) Department of Science and Technology, Graduate School of Medicine, Science and Technology, Shinshu University, Matsumoto, Nagano, 390-8621, Japan E-mail address: yazawa@math.shinshu-u.ac.jp 